\title{
Efficient Routing Strategy with Memory Information for Complex Networks
}

\author{
Takayuki Kimura ${ }^{1}$, Tohru Ikeguchi ${ }^{2}$, Chi K. Tse ${ }^{3}$ \\ ${ }^{1}$ Department of Electrical and Electronic Engineering, Nippon Institute of Technology, Saitama, Japan \\ ${ }^{2}$ Graduate School of Science and Engineering, and Saitama University Brain Science Institute, Saitama University, Saitama, Japan \\ ${ }^{3}$ Department of Electronic and Information Engineering, Hong Kong Polytechnic University, Hong Kong, China \\ Email: tkimura@nit.ac.jp
}

Received November 17, 2011; revised December 20, 2011; accepted December 30, 2011

\begin{abstract}
In this paper, we propose a new packet routing strategy that incorporates memory information for reducing congestion in communication networks. First, we study the conventional routing strategy which selects the paths for transmitting packets to destinations using the distance information and the dynamical information such as the number of accumulating packets at adjacent nodes. Then, we evaluate the effectiveness of this routing strategy for the scale-free networks. From results of numerical simulations, we conclude that this routing strategy is not effective when the density of the packets increases due to the impermeability of the communication network. To avoid this undesirable problem, we incorporate memory information to the routing strategy. By using memory information effectively, packets are spread into the communication networks, achieving a higher performance than conventional routing strategies for various network topologies, such as scale-free networks, small-world networks, and scale-free networks with community structures.
\end{abstract}

Keywords: Congestion Control; Packet Routing Problems; Decentralized Control; Complex Networks

\section{Introduction}

As a result of the rapid expansion of the Internet and associated applications such as World-Wide-Web, increasing volume of data traffic is required to be carried by communication networks. Appropriate transmission protocols are mandatorily used to optimize the carried data traffic. It has been shown that the shortest path protocol commonly employed by communication networks is facing serious challenge if the data volume continues to increase [1]. Specifically, the shortest path protocol transmits data using only the distance information of the network, and the routers where a large number of shortest paths go through are easily congested. Thus, it is imperative to enhance the transmission algorithm in order to ensure reliable communication through the network. In this connection, an understanding of the data flow dynamics of the packets would be necessary. For example, Ohira et al. [2] studied the optimal network structure for packet flow in communication networks, and they showed the onset of phase transition behavior from free-flow state to congestion state as the packet creation rate increases. Arenas et al. [3] analyzed the phase transition behavior for hierarchical branching networks. Zhao et al. [4] studied the dynamics of flowing packets for various network topologies, and showed that the networks with significant heterogeneous property would inhibit packet congestion.

To improve the capability of the network in carrying a large volume of data traffic, we need effective routing strategies which can reduce drastically the congestion of the network. Recent works in the development of routing strategies have evolved along two basic ideas. The first one is the selection of paths for transmitting packets based on only local information of the network such as degree information [5,6], and/or the number of packets waiting at adjacent nodes [7]. These routing strategies can be applied to networks of any size because collection of the distance information of the whole network is not necessary. The second idea is to utilize global information such as the shortest distance information of the network. For example, Echenique et al. [1,8] proposed a deterministic routing strategy, which calculates the efficient paths of sending packets by distance information and dynamic information. Yan et al. [9] proposed a routing strategy using both the distance information and the degree information in the network. Zhang et al. [10] proposed an adaptive routing strategy based on measurement of the accumulated number of packets on the shortest paths. A routing strategy with traffic awareness was proposed by Wang et al. [11]. Also, Horiguchi et al. [12] proposed a routing strategy employing mutual con- 
nected neural networks. This method was further improved by reinforcement of a learning strategy [13] and incorporation of stochastic effects [14].

In this paper, we study the routing strategy along the idea of using global network information, and we propose a new approach to reduce congestion by incorporating memory information. First, we construct a routing strategy which selects the paths for transmitting the packets using the distance information and the dynamical information such as the number of accumulating packets in the network. We evaluate this conventional routing strategy for scale-free network. It is shown that due to the impermeability of the network [8], this routing strategy performs poorly as the density of the packets increases. To overcome this problem, we introduce the use of memory information in the routing strategy. It can be shown that memory information such as the amount of packets sent in adjacent nodes can be exploited to avoid congestion substantially. Numerical simulations show that the proposed routing strategy achieves higher performance than the conventional routing strategies for various network topologies, including scale-free networks [15], small-world networks [16], and scale-free networks with community structures [17].

This paper is organized as follows. In Section 2, we describe a communication network model. In Section 3, we consider the conventional type of routing strategy that uses distance information and dynamical information, and evaluates the performance of this routing strategy for scale-free networks. The cause for poor performance as the density of the packets increases is identified. In Section 4 , we introduce the use of memory information in the routing strategy, and evaluate the effectiveness of the proposed routing strategy in Section 5. We conclude the paper in Section 6.

\section{Communication Network Model}

We take an unweighted and undirected graph $G=(V, E)$ as the network model, where $V$ is the set of nodes and $E$ is the set of links. Each node represents a host and a router in the network, and each link represents a physical connection between two nodes. If a packet is created at a node, the packet is stored at the tail of the buffer of the node. In addition, a packet at the head of the buffer of the node is transmitted to an adjacent node. In other words, all the packets are transmitted to their destinations according to the first-in-first-out principle. Sources and destinations of the packets are randomly selected using uniformly distributed random numbers. In addition, if a node to which a packet will be transmitted has a full buffer, movement of the packet is cancelled and the packet must wait for the next opportunity to be transmitted in the following step. To construct realistic commu- nication networks, we assign to each node a largest storage capacity and a processing capacity [5].

The largest storage capacity corresponds to the maximum size of the buffer that stores the packets, and the processing capability corresponds to the maximum number of transmitting packets at a time.

The largest storage capacity of the $i$ node, $B_{i}$, is defined as

$$
B_{i}=\rho k_{i}
$$

where, $\rho>0$ is a controlling parameter and $k_{i}$ is the degree of the $i$ th node. The largest storage capacity is proportional to the degree of the node. In other words, hub nodes in the network have a large memory to store packets. The processing capability of the $i$ th node, $C_{i}$, is defined as

$$
C_{i}=\lambda B_{i}
$$

where $\lambda<0 \leq 1$ is a controlling parameter.

If $B_{i}$ and $C_{i}(i=1, \cdots, N)$ are set to large values, congestion of packets hardly occurs because each node has much capacity for storing and transmitting the packets. However, the cost of such a communication network is very high. Thus, it is desirable to develop a packet routing strategy that works well with small values of $B_{i}$ and $C_{i}$. From this viewpoint, we set $\rho$ and $\lambda$ in Equations (1) and (2) to 2.0 and 0.2, respectively [5].

\section{Evaluation of the Routing Strategy for Scale-Free Networks}

First, we consider the routing strategy that estimates the paths for transmitting the packets based on the distance information and the dynamical information, such as the number of accumulating packets at the adjacent nodes. In this routing strategy, the shortest path length from the ith node to a destination though the $j$ th adjacent node, $y_{i j}(t+1)$, is defined as

$$
y_{i j}(t+1)=\xi_{i j}(t+1)
$$

where,

$$
\begin{aligned}
\xi_{i j}(t+1)= & \beta\left\{H\left(\frac{d_{i j}+d_{j g\left(p_{i}(t)\right)}}{\sum_{k=1}^{N_{i}}\left(d_{i k}+d_{k g\left(p_{i}(t)\right)}\right)}\right)\right. \\
& \left.+(1-H)\left(\frac{q_{j}(t)}{\sum_{k=1}^{N_{i}} q_{k}(t)}\right)\right\}
\end{aligned}
$$

with $\beta$ being a controlling parameter, $N_{i}$ being the number of the adjacent nodes of the ith node, $p_{i}(t)$ being a packet transmitted from the $i$ th node at the $t$ th time, $g\left(p_{i}(t)\right)$ being the destination of $p_{i}(t), d_{i j}$ the static distance between the $i$ th node and the $j$ th adjacent node, $d_{j g\left(p_{i}(t)\right)}$ the shortest distance between the $j$ th adjacent 
node and $g\left(p_{i}(t)\right)$, and $q_{j}(t)$ the number of accumulating packets of the $j$ th adjacent node at the th time.

We note that in Equation (4), the first term expresses the distance from the ith node to the destination of the packet through the $j$ th adjacent node and the second term expresses the number of packets that are being accumulated at the $j$ th adjacent node. If Equation (3) of the $j$ th adjacent node takes the smallest value among all the $N_{i}$ adjacent nodes, i.e., the $j$ th adjacent node is the closest to the destination of the packet and has the smallest number of accumulated packets in its buffer, a packet at the ith node is transmitted to the $j$ th adjacent node. The routing strategy with $H=1$ is same as the one used in the real communication network, i.e., the packets are transmitted to the destinations using the distance information only. For ease of distinction, we refer to this routing strategy with $H=1$ as standard routing strategy. If $H<1$, the routing strategy uses not only the distance information but also the dynamical information of the numbers of accumulating packets at the adjacent nodes. We call such a routing strategy a gain routing strategy. Although the deterministic protocol proposed by Echenique et al. $[1,8]$ uses the same information, both the distance information and the dynamical information of the number of accumulating packets at the adjacent nodes, for routing packets, the difference between the deterministic protocol $[1,8]$ and the gain routing strategy is that Equation (4) is normalized in the case of the gain routing strategy and the deterministic protocol uses the direct information $[1,8]$.

Here we evaluate the gain routing strategy for scalefree networks. Since real communication networks are scale-free [18], we will adopt the scale-free topology as the network topology studied here. The scale-free networks are constructed by the following procedure [15]. We begin with a complete graph with four nodes. Then, we put a new node with four links at every time step. Next, we connect four links of the newly added node to the nodes that already exist in the network with probability $\Pi\left(k_{i}\right)=k_{i} / \sum_{j=1}^{N} k_{j}$, where $k_{i}$ is the degree of the $i$ th node $(i=1, \cdots, N)$, and $N$ is the number of the nodes at the current iteration.

Numerical simulations are conducted as follows. First, packets are created from randomly selected sources and destinations. Then, at every node, an optimal adjacent node was selected using Equations (3) and (4), and the packets are simultaneously transmitted to their destinations. The number of packets in the communication networks is fixed. Thus, when a packet arrives at its destination, it is removed and a new packet is created from randomly selected source and destination. We repeat the node selection and packet transmission, $I$, for $I=1000$. Also, $\beta$ in Equation (4) is set to 1.

To evaluate the performance of the gain routing strat- egy, we use the following metrics.

1) Density of the packets $(D)$ :

$$
D=p \sum_{i=1}^{N} B_{i}
$$

where $B_{i}$ is the largest storage capacity defined by Equation (1) and $(0<p \leq 1)$ is the ratio of the number of packets to the capacity of the network. If $p$ increases, a large number of packets are flowing in the network.

2) The number of flows $(F)$ :

$$
F=\sum_{t=1}^{I} f(t)
$$

where $f(t)$ is the number of packets transmitted to the adjacent nodes at the th time and $I$ is the number of iterations. If there is no packet congestion in the network, each node can transmit the packets to their destinations because the adjacent nodes can store the packets in their buffers. Thus, the number of flows $(F)$ increases.

3) Average arrival rate of the packets $(A)$ :

$$
A=\frac{N_{a}}{N_{c}}
$$

where $N_{c}$ is the total number of packets created in the network and $N_{a}$ is the total number of arriving packets. The average arrival rate $(A)$ is an important measure to evaluate the routing strategy. By reducing or inhibiting the packet congestion in the network, the routing strategy can maintain a higher arrival rate $(A)$.

4) Average arrival time of the packets $(T)$ :

$$
T=\frac{1}{N_{r}} \sum_{i=1}^{N_{r}} T(i)
$$

where $N_{r}$ is the number of packets to record the arrival times and $T_{i}$ is an arrival time of the ith packet. In Equation (8), if the ith packet cannot be transmitted to its destination during the simulation, the arrival time of the $i$ th packet, $T_{i}$, will be assigned as $T_{i}=I$. We set $N_{r}$ to 1000 . If the arrival rate $(T)$ becomes small as a result of the routing strategy, packets can be sent to their destinations very quickly.

Results for scale-free networks of 100 nodes are shown in Figure 1. In Figure 1(a), in the case of the standard routing strategy $(H=1$ in Equation (4)), the number of flows $(F)$ rapidly decreases when the density of the packets $(D)$ is larger than 10 . This result indicates that congestion of the packets occurs at $D=10$, and the packets cannot be transmitted to the adjacent nodes. As a result, the value of $F$ of the standard routing strategy decreases. The gain routing strategy with $H=0.2$ and the one with $H=0.3$ give larger values of $F$ than with other $H$ values. Also, the gain routing strategy with $H=0.3$ has a higher arrival rate of the packets $(A)$ than with other $H$ values, as shown in Figure 1(b). Furthermore, the gain 


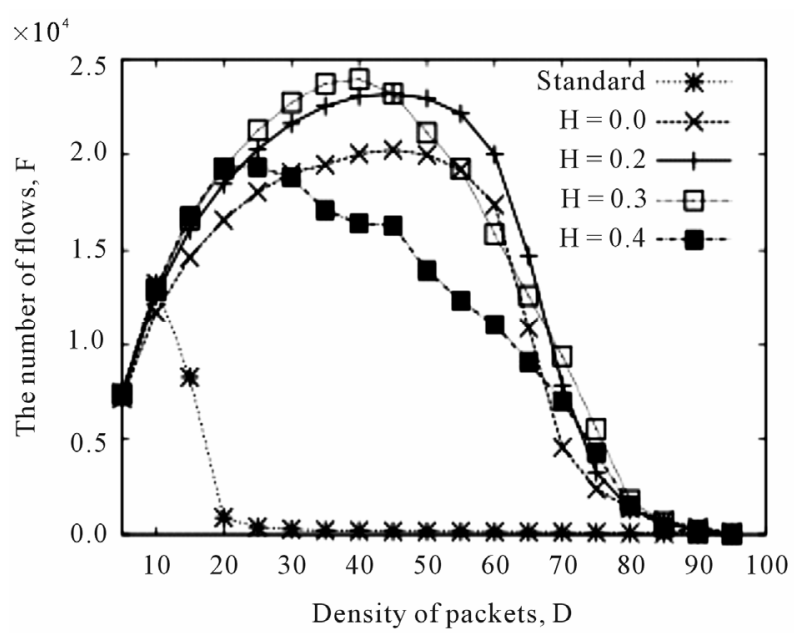

(a)

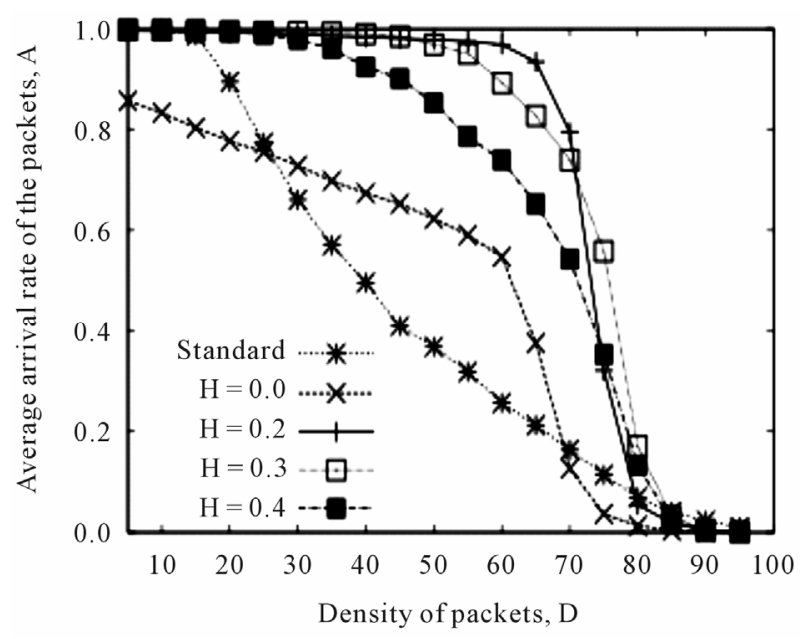

(b)

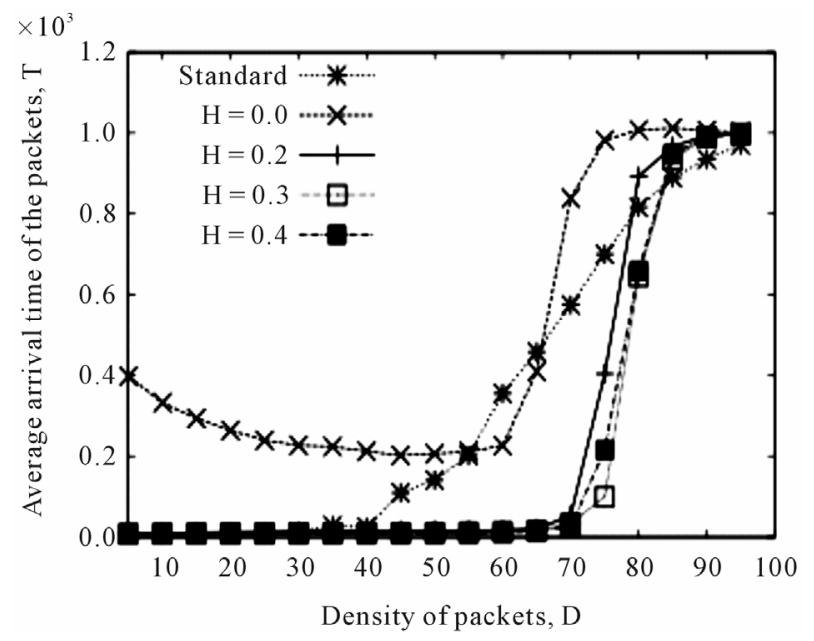

(c)

Figure 1. Relationship between the density of the packets $(D)$ and (a) Number of flows (F); (b) Arrival rate of the packets $(A)$; and (c) Average arrival time of the packets ( $T$ ) for scale-free networks. routing strategy with $H=0.3$ transmits packets faster than with other $H$ values, as shown in Figure 1(c). Thus, from Figure 1, we may conclude that the gain routing strategy with $H=0.3$ gives the best performance for scale-free networks.

However, we can see that the number of flows $(F)$ for all cases rapidly decreases when the density of the packets $(D)$ becomes large. Also, the average arrival time $(T)$ becomes large as the density of the packets $(D)$ increases. Thus, to probe further, we measure the congestion levels of the nodes for the scale-free networks. The congestion level of the $i$ th node can be found using $q_{i}(t) / B_{i}$ $\left(0 \leq q_{i}(t) / B_{i} \leq 1\right)$. If $q_{i}(t) / B_{i}$ takes 1 , no adjacent nodes can transmit the packets to the ith node at the $t$ th iteration because the ith node has a full buffer. The congestion levels of the nodes by the gain strategy with $H=$ 0.3 are shown in Figure 2. Here, we see that although the congestion levels of the nodes show similar values when the density of the packets $(D)$ is 10 (see Figure 2(a)), the nodes show higher congestion values when $D$ is 70 , as shown in Figure 2(b). Further, in Figure 2(b), we can see that the congestion levels have two separate states: non-congested states (green) and congested states (red). Moreover, once the state of the node changes, it remains unchanged throughout the simulation.

To understand this phenomenon, we consider the following typical situation [8]. The ith node has a packet for destination node $g$. In this case, the $i$ th node has two adjacent nodes, i.e., node $j_{1}$ and node $j_{2}$. Then, we assume that for node $j_{1}$, the second term of (4) has a smaller value than that of node $j_{2}$. In addition, node $j_{1}$ needs to take two hops to reach node $g$, while node $j_{2}$ needs to take one hop to reach node $g$. In other words, node $j_{2}$ is closer to the destination $g$ than node $j_{1}$. However, the packet cannot be transmitted to node $j_{2}$ if following relation is not satisfied:

$$
\begin{aligned}
& \left(q_{j 1}(t) / \sum_{k=1}^{2} q_{k}(t)-q_{j 2}(t) / \sum_{k=1}^{2} q_{k}(t)\right) \\
& >H /\{5(1-H)\}
\end{aligned}
$$

Thus, node $j_{2}$ becomes impenetrable for the ith node in this case.

Figure 3 shows the percentage of the available paths in the network for the gain strategy with $H=0.3$. Although $100 \%$ of the available paths are used for routing the packets when the density of the packets $(D)$ is 10 , the number of available paths decreases to $60 \%$ when $D$ is 70. These results indicate that if the number of impenetrable nodes increases in the network, the number of available paths decreases. This phenomenon is known as impermeability of the network which was originally studied by Echenique et al. [8]. We also see that although there are some non-congested nodes when $D$ is 70 as shown in Figure 2(c), the arrival rate of the packets is 


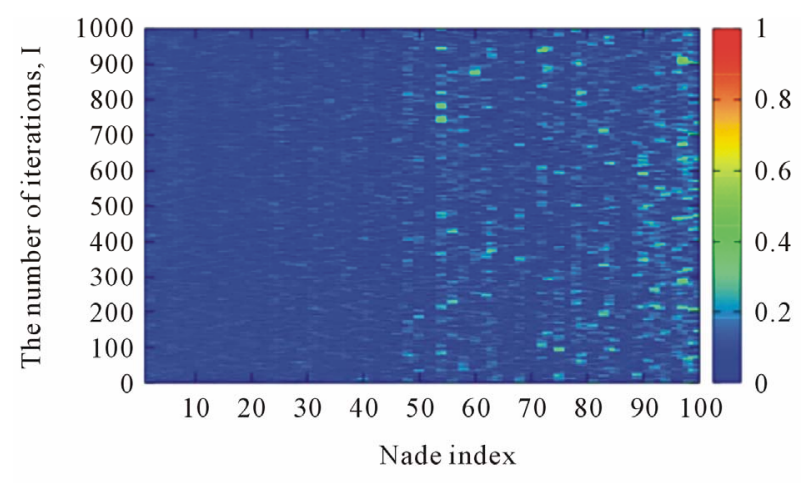

(a)

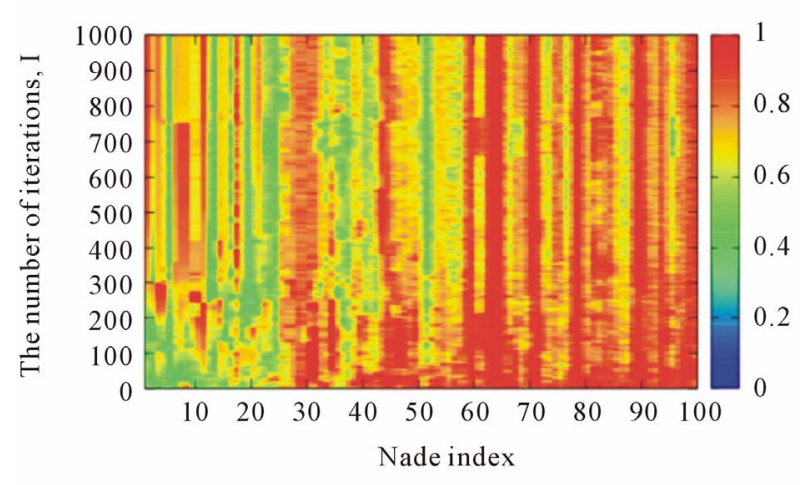

(b)

Figure 2. Congestion levels of the nodes as a function of the number of iterations $(I)$ and the node index by the gain routing strategy with $H=0.3$ when (a) the density of the packets $(D)$ is 10 , and (b) $D=70$.

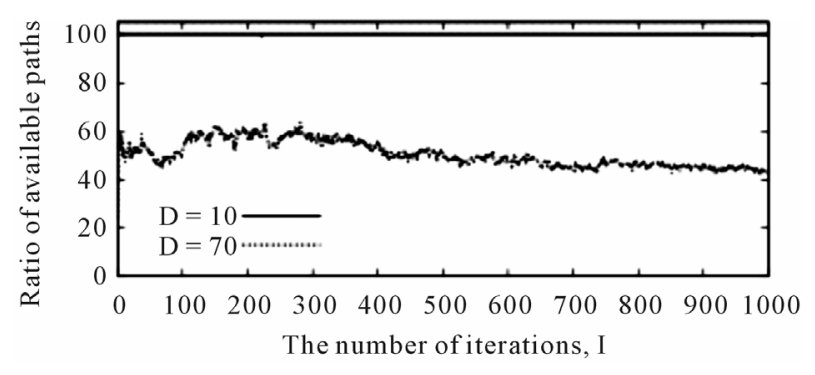

Figure 3. Relationship between the percentage of the available paths and the number of iterations ( $I$ for the gain routing strategy with $H=0.3$.

much lower, as given in Figure 1(b). This is because there are a small number of available paths for transmitting the packets in the network.

\section{Packet Routing Strategy with Memory Information}

In Section 3, we have shown that the gain routing strategy becomes ineffective when the density of the packets becomes large due to the impermeability of the network. It is thus imperative to develop effective routing strategies to overcome the impermeability problem. Intuitively, an effective routing strategy should avoid sending packets to nodes to which many packets have already been transmitted in the past routings. In this work, we introduce the use of memory information in the gain routing strategy [19]. Specifically, we modify Equation (3) as follows:

$$
y_{i j}^{\prime}(t+1)=\xi_{i j}(t+1)+\zeta_{i j}(t+1)
$$

where $\xi_{i j}(t+1)$ has been defined in Equation (4), i.e.,

$$
\zeta_{i j}(t+1)=\alpha \sum_{\gamma=0}^{t} k_{r}^{\gamma} x_{i j}(t-\gamma)=\alpha x_{i j}(t)+k_{r} \zeta_{i j}(t-1)
$$

with $\alpha$ being a scaling parameter of the memory information, $k_{r}$ a decay parameter, and $x_{i j}(t)$ the transmission history of the $j$ th adjacent node at the th time, i.e.,

$$
x_{i j}(t)=\left\{\begin{array}{cc}
1 & \left(\min \left(y_{i j}^{\prime}(t+1)\right)\right) \\
0 & \text { (otherwise) }
\end{array}\right.
$$

If $y_{i j}^{\prime}(t+1)$ of the $j$ th adjacent node takes the smallest value among all the $N_{i}$ adjacent nodes, a packet at the $i$ th node is transmitted to the $j$ th adjacent node. Then, the transmission history of the $j$ th adjacent node, $x_{i j}(t)$, is updated according to Equation (11).

\section{Numerical Simulations for Various Complex Networks}

In this section we evaluate the performance of the proposed routing strategy for different network topologies. In particular, since existing communication networks exhibit scale-free and small-world properties, it is of interest to consider scale-free networks [15], small-world networks [16], and scale-free networks with community structures [17]. In our simulations, we compare the proposed routing strategy with two conventional routing strategies. The first one is the standard routing strategy described in Section 3, which is realized by setting $H$ to 1.0. The second one is the gain routing strategy with $H=$ 0.3. We set the parameters in Equations (4), (10) and (11) for the proposed routing strategy as follows: $\beta=3.0$, $\alpha=0.07, \quad k_{r}=0.98$.

\subsection{Scale-Free Networks}

First, we evaluate the proposed routing strategy for scalefree networks under the same experimental conditions as used in Section 3. Figure 4 shows the percentage of available paths for scale-free networks using the standard routing strategy, the gain routing strategy, and the proposed routing strategy. As shown in Figure 4, the percentage of the available paths for the proposed routing strategy is larger than those of the standard routing strategy and the gain routing strategy. This result indicates 


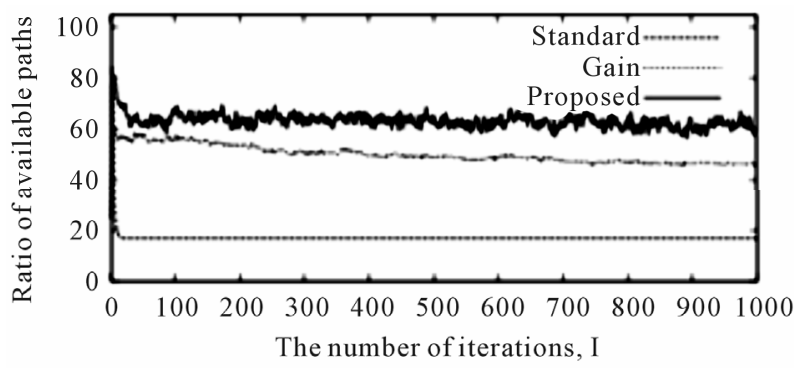

Figure 4. Relationship between the percentage of available paths and the number of iterations $(I)$ using the standard routing strategy (Standard), the gain routing strategy (Gain), and the proposed routing strategy (Proposed) for scale-free networks. The density of the packets $(D)$ is 70 .

that the impermeability of the network is eliminated as a result of the use of the memory information.

As shown in Figure 5(a), the proposed routing strategy allows a higher number of flows $(F)$ than the standard routing strategy and the gain routing strategy, when the density of the packets $(D)$ is larger than about 55 .

In addition, as shown in Figure 5(b), the proposed routing strategy shows a higher arrival rate $(A)$ than the other strategies when $D$ is larger than 50. Further, the proposed routing strategy gives a shorter arrival time when $D$ is larger than 70 . Clearly, as the impermeability of the network is eliminated in the case of the proposed routing strategy, the packets can be transmitted to the destinations using various routes even if the density of the packets becomes large in the network.

\subsection{Small-World Networks}

Next, we evaluate the performance of the routing strategies for small-world networks [16]. Small-world networks are constructed in the same manner as described in Watts and Strogatz [16]. First, $N$ nodes are put on a closed one-dimensional ring and each node is connected to its $K$-th nearest neighbors. Then, each link is randomly re-wired with probability $R_{p}$. We set $N$ and $K$ to 100 and 4 , respectively. Results for the small-world network topology are shown in Figure 6. From Figure 6(a), although the number of flows $(F)$ for the standard routing strategy is very small for the whole range of $R_{p}$, the gain routing strategy and the proposed routing strategy show higher flows, as indicated in Figures 6(b) and (c). In addition, the value of $F$ in the case of the gain strategy and the proposed strategy increases as the rewiring probability $\left(R_{p}\right)$ increases beyond 0.8 . Moreover, although the arrival rate of the packets $(A)$ for the gain strategy decreases when $D$ is larger than 70 for almost the whole $R_{p}$ range (Figure 6(e)), the proposed strategy keeps a high arrival rate $(A)$ even if $D$ increases for almost the whole range of $R_{p}$ (Figure 6(f)). Finally, from Figures 6(g), (h), and (i), we see that the packets can be transmitted to the

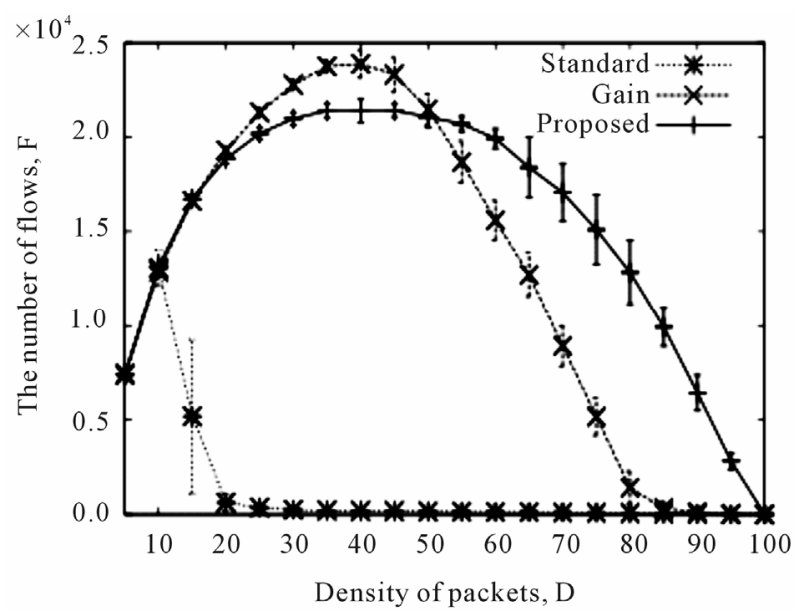

(a)

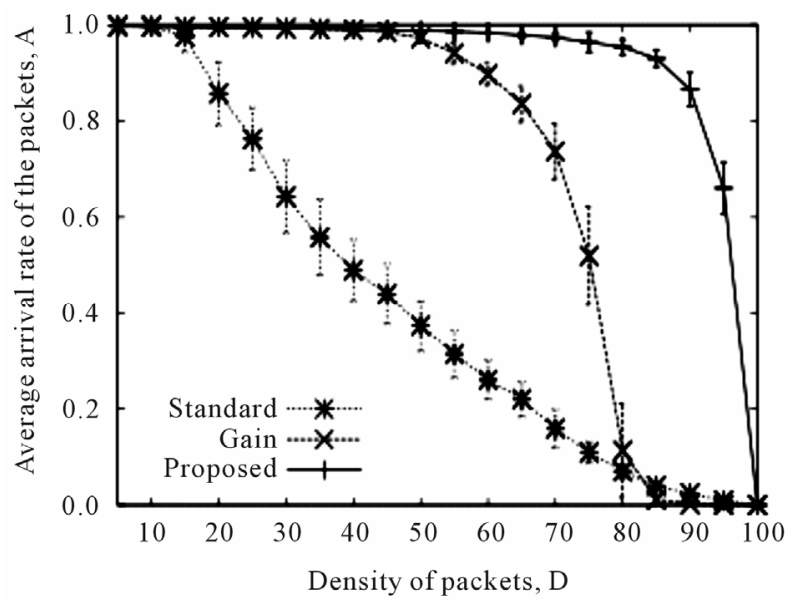

(b)

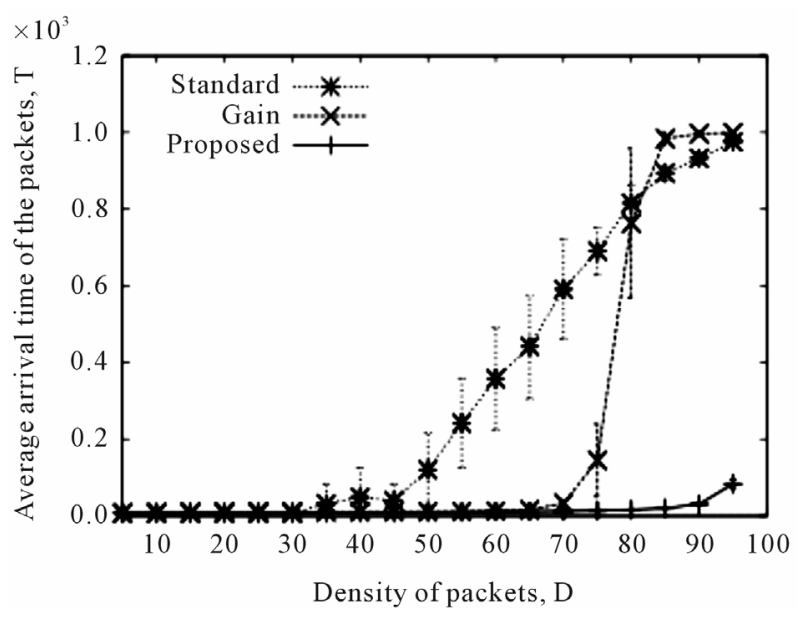

(c)

Figure 5. Relationship between the density of the packets $(D)$ and (a) The number of flows $(F)$; (b) The arrival rate of the packets $(A)$; and (c) The average arrival time of the packets (T), using the standard routing strategy (Standard), the gain routing strategy (Gain), and the proposed routing strategy (Proposed) for scale-free networks. 


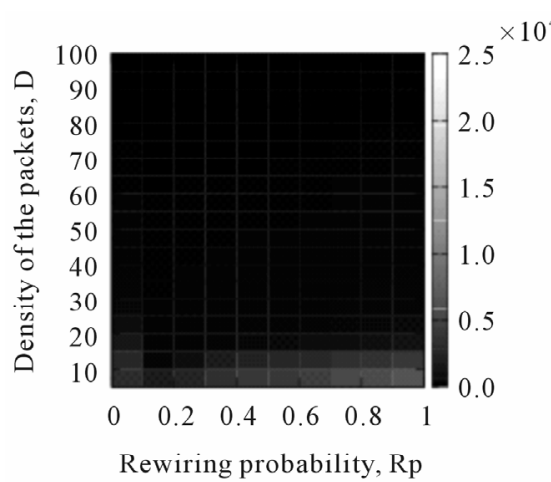

(a)

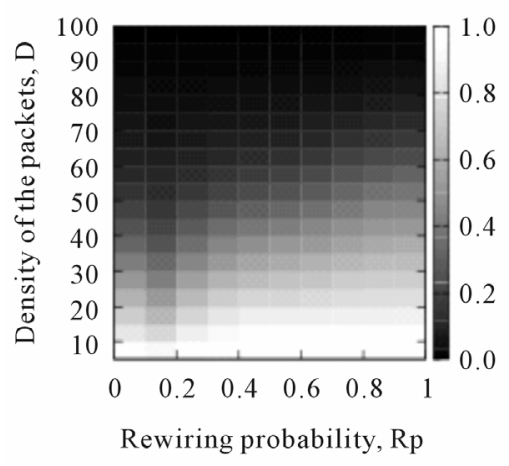

(d)

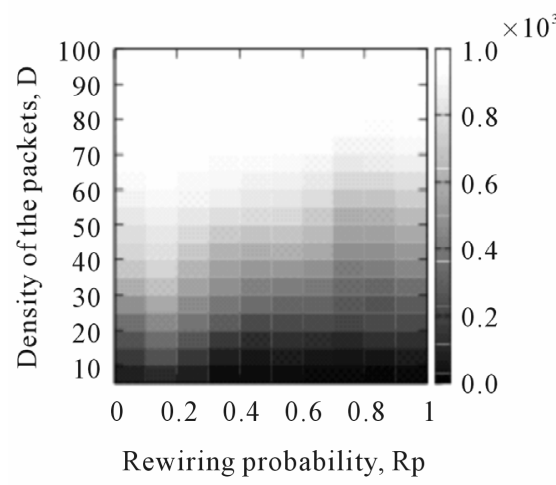

(g)

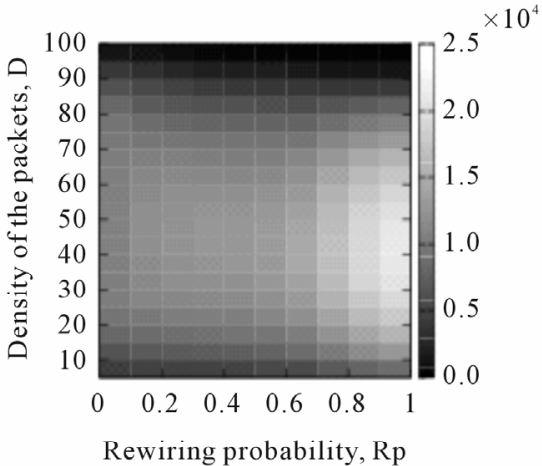

(b)

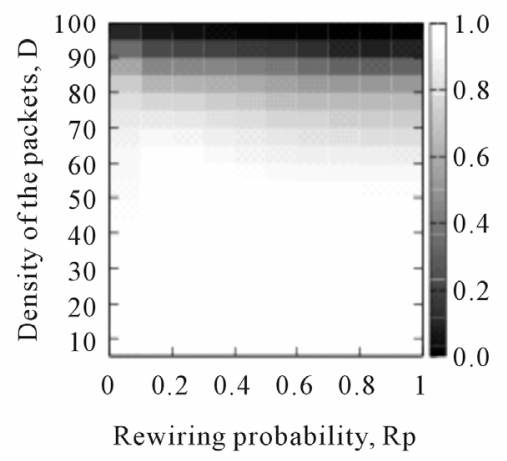

(e)

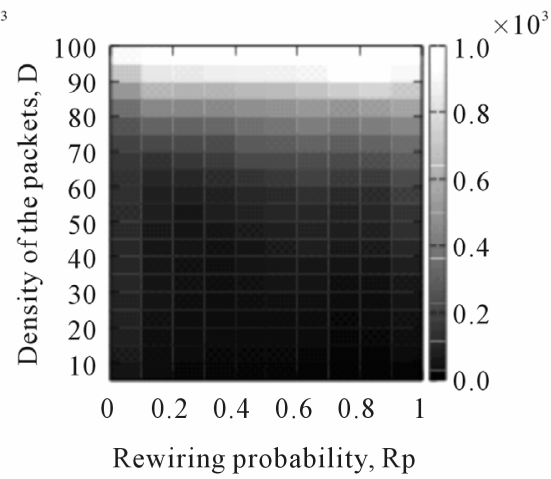

(h)

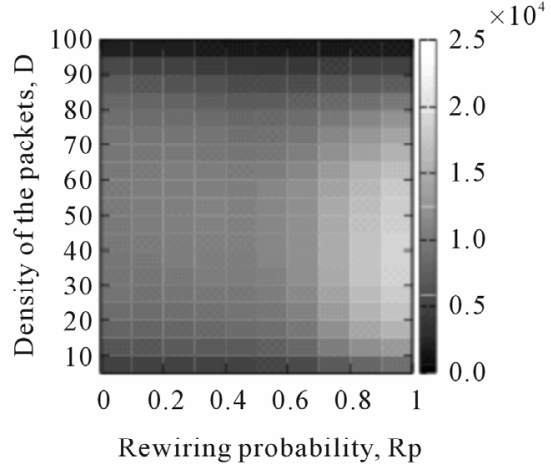

(c)

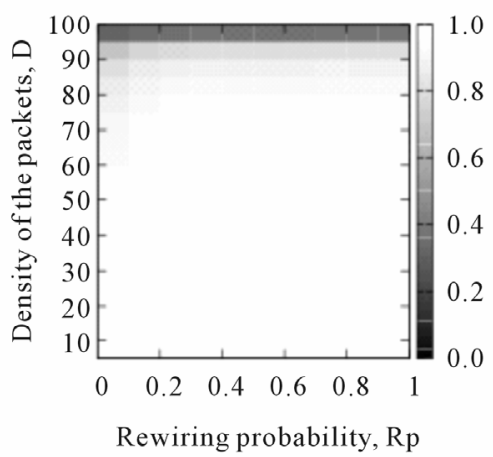

(f)

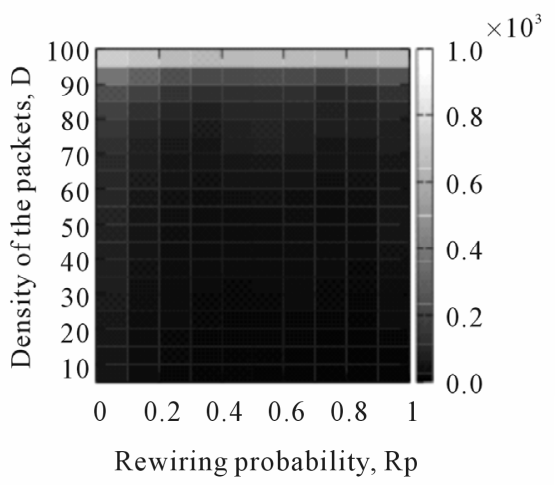

(i)

Figure 6. Relationship between the density of the packets $(D)$, the rewiring probability $\left(R_{p}\right)$, and the number of flows $(F)$ for (a) The standard strategy (Standard); (b) The gain routing strategy (Gain); and (c) The proposed routing strategy (Proposed); the average arrival rate $(A)$ for $(d)$ Standard; (e) Gain; and (f) Proposed; and the average arrival rate of the packets $(T)$ for $(g)$ Standard; (h) Gain; and (i) Proposed, for small-world networks.

destinations quickly using the proposed strategy compared to the other routing strategies for the whole range of $R_{p}$, as shown in Figure 6(f). In the case of small-world networks, the proposed routing strategy shows better performance if the topology of the network becomes more random. In a random network, there are numerous shortcuts that allow packets to be quickly transmitted to the destinations. Thus, when the network topology becomes random, the proposed routing strategy shows better performance as more shortcuts are available.

\subsection{Scale-Free Networks with Community Structures}

Finally, we evaluate the routing strategies for scale-free networks with community structures [17]. The construction procedure follows the one described in Wu et al. [20]. We begin with $C$ complete graphs, each of which forms a community of $m$ nodes. Then, we add a new node with $m$ links to each community at every time step. In each step, $m-n$ links of the newly added node are con- 
nected to the nodes in the same community, and $n$ links are connected to the nodes in the other communities. The nodes in the same community and the ones in the other communities are selected by the principle of preferential attachment. In the simulations, we set $m=4$ and $n=1$.

Results for scale-free networks with community structures of 100 nodes are shown in Figure 7. Although the number of flows $(F)$ decreases with increasing number of communities $(C)$ in the case of the proposed routing strategy, as shown in Figures 7(a), (b), and (c), either a higher arrival rate $(A)$ can be achieved, as shown in Figure $\mathbf{7 ( d )}$, or similar performance as the gain routing strategy can be achieved, as shown in Figures 7(e) and (f). These results indicate that the proposed strategy effectively selects the paths for routing packets when $C$ increases. Thus, even if $F$ decreases, the proposed strategy is able to maintain a high $A$. Figures $\mathbf{7 ( g ) , ~ ( h ) , ~ a n d ~}$ (i) show that the proposed strategy transmits the packets much faster than the other strategies even if $C$ increases. Thus, we may conclude that in the case of scale-free networks with community structures, the proposed routing strategy can effectively route the packets to the destinations, and is able to transmit the packets quickly to their destinations even if the number of communities is large.

\section{Conclusions}

In this paper, we study the routing strategies in communication networks exploiting the use of global information, and in particular we incorporate memory information to select paths for transmitting packets that can eliminate traffic congestion. We first examined the routing strategy that uses the distance information and the dynamical information, and evaluated its performance for the scale-free networks. It has been shown that such a

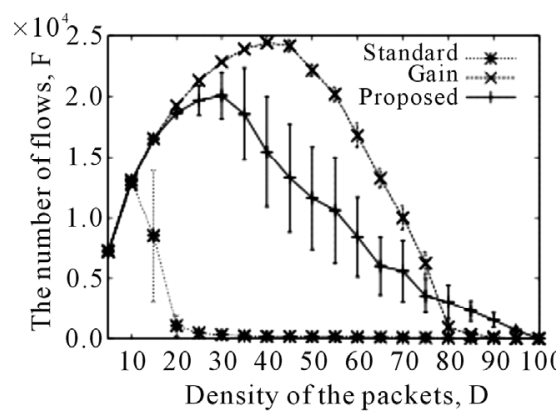

(a)

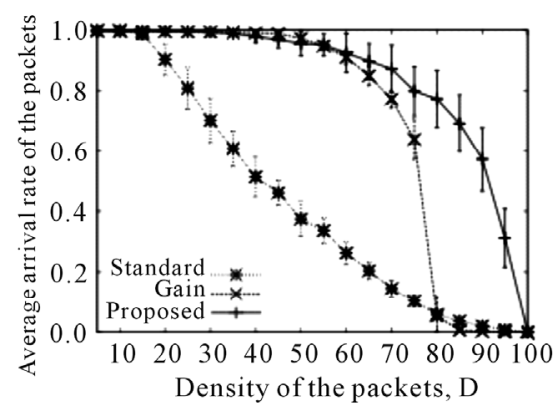

(d)

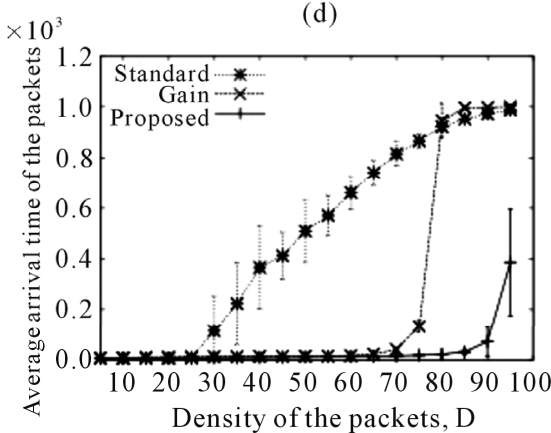

(g)

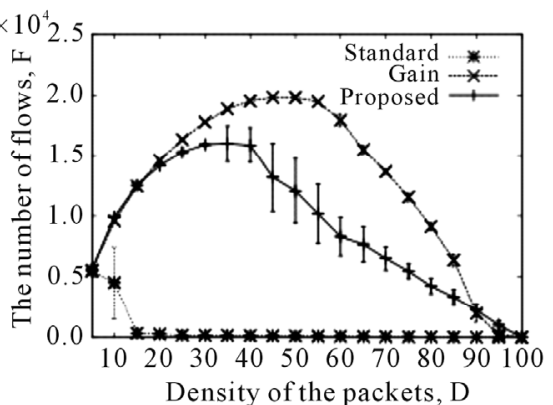

(b)

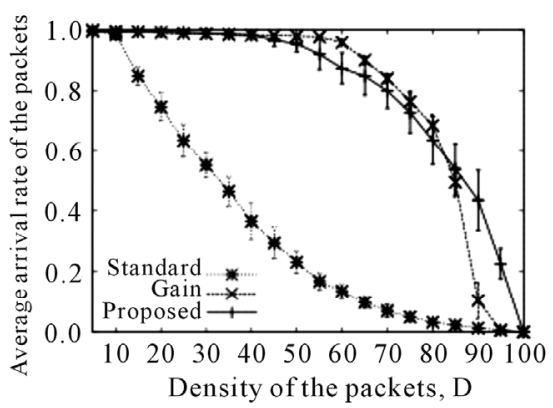

(e)

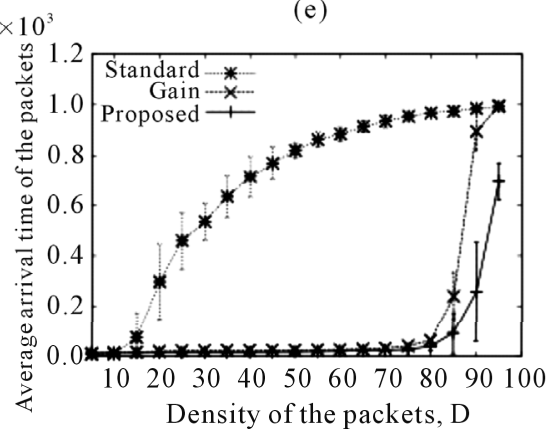

(h)

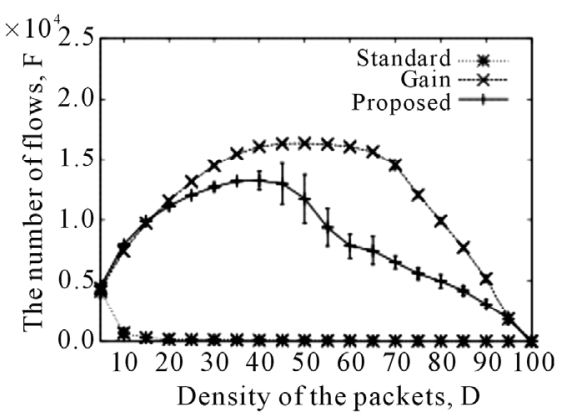

(c)

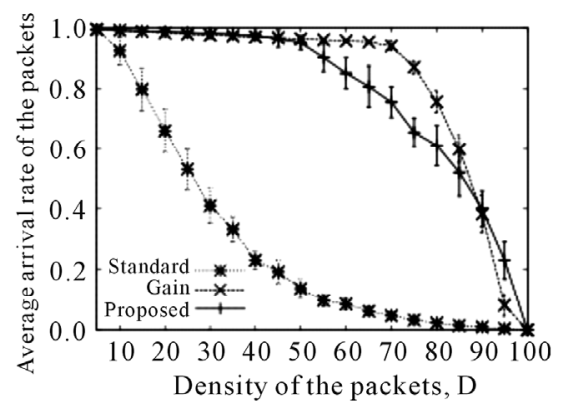

(f)

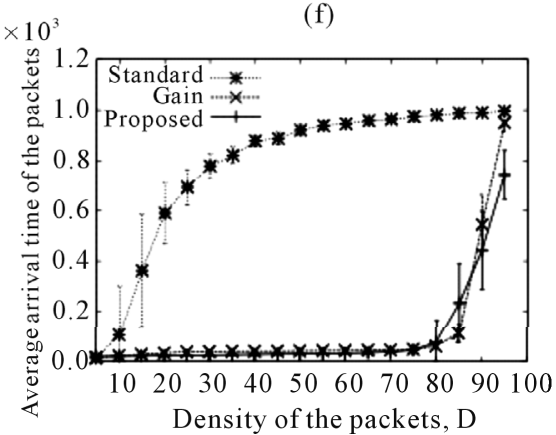

(i)

Figure 7. Relationship between the density of the packets $(D)$ and the number of flows $(F)$ for $(a)$ the number of communities $C=2$; (b) $C=10$; and (c) $C=15$; the average arrival rate $(A)$ for (d) $C=2$; (e) $C=10$; and (f) $C=15$; and the average arrival time of the packets $(A)$ for (g) $C=2$; (h) $C=10$; and (i) $C=15$, for scale-free networks with community structures. 
routing strategy becomes ineffective if the density of the packets increases due to the impermeability of the network that results in reduced number of paths available for routing. To avoid network impermeability, we have proposed the inclusion of memory information in the routing strategy. By using the memory information effectively, we maintain a large number of available paths even if the density of the packets becomes high. As a result, the proposed routing strategy shows better performance than the conventional routing strategies for various network topologies such as scale-free networks, small-world networks [16] and scale-free networks with community structure [17].

In our future work, the use of memory information may be combined with consideration of the use of cellular automata for congestion elimination. In evaluating the performance of routing strategies, an order parameter may be used to indicate the phase transition point between free state and congested state for the network under study [1,8-11]. A novel evaluation method may be proposed for routing strategies using the order parameter.

The research of T. K. was partially supported by Grantin-Aid for Young Scientists (B) from JSPS (No. 23700180).

\section{REFERENCES}

[1] P. Echenique, J. Gómez-Gardenes and Y. Moreno, "Improved Routing Strategies for Internet Traffic Delivery," Physical Review E, Vol. 70, No. 5, 2004, Article ID: 056105, pp. 1-4. doi:10.1103/PhysRevE.70.056105

[2] T. Ohira and R. Sawatari, "Phase Transition in A Computer Network Traffic Model,” Physical Review E, Vol. 58, No. 1, 1998, pp. 193-195. doi:10.1103/PhysRevE.58.193

[3] A. Arenas, A. Díaz-Guilera and R. Guimerà, "Communication in Networks with Hierarchical Branching," Physical Review Letters, Vol. 86, No. 14, 2002, pp. 3196-3199. doi:10.1103/PhysRevLett.86.3196

[4] L. Zhao, Y.-C. Lai, K. Park and N. Ye, "Onset of Traffic Congestion in Complex Network," Physical Review E, Vol. 71, No. 2, 2005, Article ID: 026125, pp. 1-8. doi:10.1103/PhysRevE.71.026125

[5] M.-B. Hu, W.-X. Wang, R. Jiang, Q.-S. Wu and Y.-H. $\mathrm{Wu}$, "Phase Transition and Hysteresis in Scale-free Network Traffic, Physical Review E, Vol. 75, No. 3, 2007, Article ID: 036102, pp. 1-4. doi:10.1103/PhysRevE.75.036102

[6] W.-X. Wang, B.-H. Wang, C.-Y. Yin, Y.-B. Xie and T. Zhou, "Traffic Dynamics Based on Local Routing Protocol on a Scale-Free Network,” Physical Review E, Vol. 73, No. 2, 2006, Article ID: 026111, pp. 1-7. doi:10.1103/PhysRevE.73.026111
[7] W.-X. Wang, C.-Y. Yin, G. Yan and B.-H. Wang, "Integrating Local Static and Dynamic Information for Routing Traffic,” Physical Review E, Vol. 74, No. 1, 2006, Article ID: 016101, pp. 1-5. doi:10.1103/PhysRevE.74.016101

[8] P. Echenique, J. Gómez-Gardenes and Y. Moreno, "Dynamics of Jamming Transitions in Complex Networks," Europhysics Letters, Vol. 71, No. 2, 2005, pp. 325-331. doi:10.1209/epl/i2005-10080-8

[9] G. Yan, T. Zhou, B. Hu, Z.-Q. Fu and B.-H. Wang, "Efficient Routing on Complex Networks,” Physical Review E, Vol. 73, No. 4, 2006, Article ID: 046108, pp. 1-5. doi:10.1103/PhysRevE.73.046108

[10] H. Zhang, Z. Liu, M. Tnag and P. Hui, “An Adaptive Routing Strategy for Packet Delivery in Complex Networks,” Physics Letters A, Vol. 364, No. 3-4, 2007, pp. 177-182. doi:10.1016/j.physleta.2006.12.009

[11] D. Wang, Y. Jing and S. Zhang, “Traffic Dynamics Based on A Traffic Awareness Routing Strategy on Scale-Free Networks,” Physica A, Vol. 387, No. 12, 2008, pp. 30013007. doi:10.1016/j.physa.2008.01.085

[12] T. Horiguchi and S. Ishioka, "Routing Control of Packet Flow Using A Neural Network," Physica A, Vol. 297, No. 3-4, 2001, pp. 521-531. doi:10.1016/S0378-4371(01)00229-1

[13] T. Horiguchi, K. Hayashi and A. Tretiakov, "Reinforcement Learning for Congestion-Avoidance in Packet Flow,” Physica A, Vol. 349, No. 1-2, 2005, pp. 329-348. doi:10.1016/j.physa.2004.10.015

[14] T. Kimura, H. Nakajima and T. Ikeguchi, “A Packet Routing Method for Complex Networks by A Stochastic Neural Network,” Physica A, Vol. 376, 2007, pp. 658-672. doi:10.1016/j.physa.2006.10.061

[15] A.-L. Barabási and R. Albert, "Emergence of Scaling in Random Networks,” Science, Vol. 286, No. 5439, 1999, pp. 509-512. doi:10.1126/science.286.5439.509

[16] D. Watts and S. Strogatz, "Collective Dynamics of SmallWorld Networks,” Nature, Vol. 393, 1998, pp. 440-442. doi:10.1038/30918

[17] M. Newman and M. Girvan, "Finding and Evaluating Community Structure in Networks,” Physical Review E, Vol. 69, No. 2, 2004, Article ID: 026113, pp. 1-15. doi:10.1103/PhysRevE.69.026113

[18] M. Faloutsos, F. Faloutsos and C. Faloutsos, "On PowerLaw Relationships of the Internet Topology,” ACM SIGCOMM Computer Communication Review, Vol. 29, No. 4, 1999, pp. 251-262. doi:10.1145/316194.316229

[19] K. Aihara, T. Tanabe and M. Toyoda, "Chaotic Neural Network,” Physics Letters A, Vol. 144, No. 6-7, 1990, pp. 333-340. doi:10.1016/0375-9601(90)90136-C

[20] J.-J. Wu, Z.-Y. Gao and H.-J. Sun, “Cascade and Breakdown in Scale-Free Networks with Community Structure,” Physical Review E, Vo. 74, No. 6, 2006, Article ID: 066111, pp. 1-5. 